\title{
Diagnostic Role of Osteopontin in Interstitial Lung Disease
}

\author{
Meena Goyal, M.B.B.S. ${ }^{1}$, Shivani Jaswal, M.D. ${ }^{1}$, Kranti Garg, M.D. ${ }^{2}$, Seema Gupta, M.D. ${ }^{1}$, \\ Soma Dey, M.D. ${ }^{1}$, Kashish Dutta, M.D. ${ }^{2}$
}

'Department of Biochemistry, Government Medical College and Hospital, Sector 32, Chandigarh 160030, India. 2Department of Pulmonary Medicine, Government Medical College and Hospital, Sector 32, Chandigarh 160030, India. Received 26 June 2020 • Revised 9 August 2020 • Accepted 16 August 2020 • Published online 11 January 2021

\begin{abstract}
:
Objective: Early diagnosis of interstitial lung disease (ILD) is desirable not only to better define pathobiological mechanisms, but also to customize treatment protocols. As osteopontin (OPN) expression may be linked to the fibrotic remodelling in ILD, this present study was planned to evaluate the usefulness of its estimation in serum for the diagnosis of ILD. Material and Methods: Serum OPN levels were estimated by enzyme-linked immunosorbent assay in 52 diagnosed cases of ILD, that were then compared with 46 patients with chronic obstructive lung disease and 46 apparently healthy controls.
\end{abstract}

Results: Median levels of serum OPN were found to be significantly higher in patients with ILD, 2.186 nanograms per milliliter $(\mathrm{ng} / \mathrm{ml})$, as compared to patients with chronic obstructive lung disease and healthy controls (1.687 and 1.923 $\mathrm{ng} / \mathrm{ml}$, respectively). The best cutoff levels of OPN, for diagnosis of ILD, was found to be $>1.08 \mathrm{ng} / \mathrm{ml}$; with a sensitivity of $88.4 \%$ and specificity of $32.6 \%$. Of the various subtypes, serum OPN levels were found to be highest in patients with idiopathic pulmonary fibrosis and interstitial pneumonia with autoimmune features; although the difference in levels between various subgroups was not found to be statistically significant ( $p$-value=0.495).

Conclusion: Serum concentration of OPN was found to be increased in patients with ILD and may be used to aid diagnosis. This opens new avenues for future research in patients with ILD, not only for the validation of the diagnostic abilities of OPN, but also as a potential target in its treatment.

Keywords: diagnostic marker, interstitial lung disease, osteopontin

Contact: Prof. Shivani Jaswal, M.D.

Department of Biochemistry, Government Medical College and Hospital,

Sector 32, Chandigarh 160030, India.

E-mail: shivanirishiraj@yahoo.co.in

This is an open access article under the CC BY-NC-ND license

(http://www.jhsmr.org/index.php/jhsmr/about/editorialPolicies\#openAccessPolicy).

J Health Sci Med Res 2021;39(3):181-189 doi: 10.31584 /jhsmr.2021780 www.jhsmr.org 


\section{Introduction}

Interstitial lung disease (ILD) is a group of lung diseases affecting the parenchymal tissue, characterized by inflammation and fibrotic infiltration of the alveolar septa. As a result of which, there is extensive tissue destruction leading to altered lung function; such as, restricted ventilation, hypoxemia, shortness of breath, exercise limitation and ultimately death from respiratory failure. ${ }^{1,2}$

A large number of diffuse parenchymal lung disorders are grouped together as ILD, because of their common clinical, radiological and histopathological features. ${ }^{3}$ ILD is composed of more than 200 recognized entities, classified into subtypes depending on their cause. The commonly encountered subtypes are ILD due to connective tissue diseases, idiopathic pulmonary fibrosis (IPF), ILD due to inhaled substances, sarcoidosis and drug induced, among others. $^{1}$

In the diagnosis and categorization of various ILDs clinicians and researchers mainly depend on clinical data; for example, history and physical examination, radiological and pulmonary function tests, and histopathological patterns; these however, do not reflect the underlying pathobiological mechanisms of the disease. Moreover, these tests do not adequately sub-phenotype patients with ILD, who may have quantitatively or qualitatively different biological mechanisms responsible for their underlying disease. ${ }^{2}$

Early diagnosis of ILD, and its subtype will thus have a potential impact on treatment decisions and will definitely influence prognosis. As the burden of the disease is on the rise, there is a need felt for newer biomarkers to aid diagnosis that may facilitate the early diagnostic process and give a better characterization of patient phenotype in ILD. Although, multiple candidate molecular biomarkers have been identified in patients with ILDs, unfortunately no biomarker has been internationally accepted for widespread implementation to date. ${ }^{4}$ Various studies are investigating newer markers for diagnostic aid in patients with ILD and osteopontin (OPN) is one such recently added biomarker.

OPN is a phosphorylated glycoprotein secreted by many cells; such as, osteoclasts, activated $\mathrm{T}$ cells and activated macrophages, and has been found to have important roles in regulating cellular processes, for instance bone mineralization, tissue remodelling and immune regulation. ${ }^{5}$ It has been suggested that OPN functions as a pro-inflammatory cytokine that is responsible for the adhesion and chemotaxis of cells such as leukocytes and vascular smooth muscle cells. ${ }^{6}$ In this regard it has been suggested that OPN may play a critical role in chronic inflammatory diseases; such as multiple sclerosis, Crohn disease, other autoimmune disorders, several types of cancer, cardiovascular diseases, diabetes, obesity and kidney stone diseases. ${ }^{7-15}$

In addition, it has also been found that OPN produced by alveolar macrophages in the lungs, functions as a fibrogenic cytokine that promotes migration, adhesion and proliferation of fibroblasts in the development of bleomycin induced murine pulmonary fibrosis. ${ }^{16}$ Messenger ribonucleic acid analysis of human lung biopsies of patients with idiopathic pulmonary fibrosis have also shown that OPN is the most prominently expressed cytokine. ${ }^{17}$

Thus, OPN may have a key role in the development of lung fibrosis in ILD. Only a few studies have been reported on the association of OPN in ILD. If the role of OPN in ILD could be established then it would be useful not only in management and prognostication of the disease, but subsequently also in its pharmacotherapy.

This present study was thus undertaken to evaluate the role of OPN in the diagnosis of ILD.

\section{Material and Methods}

This cross-sectional study was conducted on 52 diagnosed patients with ILD, between January, 2018 and 
July, 2019. The sample size was calculated to be a minimum of 40 patients with ILD, depending on the yearly patient load of ILD in the hospital; as the precise documented prevalence of the disease was not available. Diagnosed patients with ILD, older than 18 years of age attending the out-patient clinic of the Department of Pulmonary Medicine during the study period were enrolled in the study. The diagnosis of ILD was made by the clinician, using a multidisciplinary approach, with the reconciliation of clinical, radiological (high resolution computed tomography) and histological (bronchoscopy or surgical lung biopsy) information, wherever required. ${ }^{9}$ Patients with history of cardiovascular disease, cancer or any other chronic illnesses not related to ILD were excluded from the study. Patients with ILD were categorized into subtypes of ILD, based on the American Thoracic Society/European respiratory Society (ATS/ERS) 2018 Classification. ${ }^{18}$ The study group was compared with both disease controls as well as apparently healthy controls. In total, 46 patients older than 18 years of age, attending the out-patient clinic of the Department of Pulmonary Medicine, who met the diagnostic criteria of the global initiative for chronic obstructive lung disease (COPD), formed the disease control group; while 46 age matched apparently healthy volunteers formed the healthy control group. ${ }^{19} \mathrm{COPD}$ was considered in any patient with signs and symptoms of dyspnea, chronic cough, chronic sputum production and post-bronchodilator ratio of forced expiratory volume in $1 \mathrm{sec}$ to forced vital capacity less than $0.70 .{ }^{19} \mathrm{All}$ of the apparently healthy volunteers enrolled had no history of ILD or any other chronic disease, and their chest radiographs did not show evidence of any respiratory disease. The study was approved by the institutional research and ethics committee, and written informed consent was taken from all participants at the time of enrolment.

Peripheral venous blood samples were collected under all aseptic precautions, from all the participants at the time of enrolment, for the measurement of OPN.
The serum was separated and stored at $-80{ }^{\circ}$ Centigrade until analysis. Serum OPN levels were estimated using commercially available enzyme linked immunosorbent assay, with specific monoclonal antibodies against OPN (Ray Bio Human Osteopontin ELISA kit), with coefficient of variation $<10.0 \%$.

The results for normally distributed data were represented as mean \pm standard deviation (S.D.), skewed data as median with interquartile range and categorical data as $n$ percent (\%). Unpaired 't' test, Mann Whitney $U$ test and chi-square tests were used for comparison between groups for normally, skewed and categorical data, respectively. Statistical comparison between the three study groups was conducted using Kruskal Wallis test. Area under the receiver operating characteristic curve (AUC) with $95.0 \%$ confidence interval, positive predictive value and negative predictive values were calculated for the optimal cutoff level of OPN. A p-value of less than 0.05 was considered as statistically significant.

\section{Results}

The study compared the serum levels of OPN amongst patients with ILD, COPD and healthy controls. The baseline charateristics of the subjects of the study groups is shown in Table 1.

There was no significant difference in the age of the subjects of the study groups. The percentage of males was found to be more in all the three groups, although this difference was significant only in the disease control group. The levels of serum OPN were found to be higher in patients with ILD; as compared to the patients of COPD and healthy controls, and the difference was found to be statistically significant ( $p$-value=0.039) (Table 2). The difference in levels of serum OPN levels between smokers, ex-smokers and non-smokers was not found to be significant in any of the three study groups. 
Based on receiver operating characteristic ( $\mathrm{ROC}$ ) curve analysis, the best cutoff levels of OPN for diagnosis of ILD was found to be $>1.08$ nanogram per mililiter $(\mathrm{ng} / \mathrm{ml}$ ), with the highest Youden's Index of 0.211 and a sensitivity of detecting ILD of $88.4 \%$ and specificity of $32.6 \%$ (Area under curve-0.614, p-value=0.023) (Figure 1). OPN levels of more than $1.089 \mathrm{ng} / \mathrm{ml}$ were found to have a positive predictive value of $42.5 \%$ and a negative predictive value $83.3 \%$ for diagnosis of ILD in the subjects of the study group.
The patients with ILD were categorized into subtypes according to the ATS/ERS 2018 classification, and the percentage in different subtypes is shown in Table 2. Serum OPN levels were found to be highest in patients with IPF and Interstitial pneumonia with autoimmune features (IPAF) as compared to other subtypes of ILD, although the difference in levels between various subgroups was not found to be statistically significant $(p-v a l u e=0.495)$ (Table 3).

Table 1 Characteristics of subjects of the study groups

\begin{tabular}{|c|c|c|c|c|}
\hline Subject characteristics & $\begin{array}{l}\text { Patients of ILD } \\
(n=52)\end{array}$ & $\begin{array}{l}\text { Patients of COPD } \\
(n=46)\end{array}$ & $\begin{array}{l}\text { Healthy controls } \\
(n=46)\end{array}$ & $\mathrm{p}$-value \\
\hline Age (years) & $59.46 \pm 8.56$ & $61.04 \pm 7.25$ & $58.24 \pm 8.56$ & 0.651 \\
\hline \multicolumn{5}{|l|}{$\operatorname{Sex}(\%)$} \\
\hline Male & $53.8 \%$ & $78.2 \%$ & $58.6 \%$ & 0.050 \\
\hline Female & $46.1 \%$ & $21.7 \%$ & $41.3 \%$ & \\
\hline BMI $\left(\mathrm{kg} / \mathrm{m}^{2}\right)$ & $24.69 \pm 3.57$ & $23.66 \pm 7.10$ & $24.36 \pm 2.24$ & 0.539 \\
\hline Smoker (\%) & $96 \%$ & $24 \%$ & $8.7 \%$ & 0.000 \\
\hline Ex-smoker (\%) & $13.4 \%$ & $58.6 \%$ & $2.1 \%$ & \\
\hline Non-smoker (\%) & $75 \%$ & $17.4 \%$ & $89.2 \%$ & \\
\hline
\end{tabular}

$\mathrm{ILD}=$ interstitial lung disease, $\mathrm{COPD}=$ chronic obstructive pulmonary disease, $\mathrm{OPN}=$ osteopontin, $\mathrm{ng} / \mathrm{ml}=$ nanograms per $\mathrm{milliliter}, \mathrm{kg} / \mathrm{m}^{2}=\mathrm{kiligrams}$ per meter squared

Table 2 Serum levels of osteopontin in the study groups

\begin{tabular}{|c|c|c|c|c|c|}
\hline \multirow{2}{*}{$\begin{array}{l}\text { Levels of osteopontin } \\
\text { (OPN } \mathrm{ng} / \mathrm{ml})\end{array}$} & \multirow{2}{*}{$\begin{array}{l}\text { Patients of } \\
\text { ILD } \\
(n=52)\end{array}$} & \multirow{2}{*}{$\begin{array}{l}\text { Patients of } \\
\text { COPD } \\
(n=46)\end{array}$} & \multirow{2}{*}{$\begin{array}{l}\text { Healthy } \\
\text { controls } \\
(n=46)\end{array}$} & \multicolumn{2}{|c|}{$\mathrm{p}$-value } \\
\hline & & & & Mann Whitney U tests & Kruskal Wallis test \\
\hline \multirow[t]{3}{*}{ Median levels of OPN $(\mathrm{ng} / \mathrm{ml})$} & 2.186 & 1.687 & 1.923 & ILD vs COPD : 0.042 & 0.039 \\
\hline & & & & ILD vs Healthy : 0.064 & $\mathrm{H}$ statistic $=6.484$ \\
\hline & & & & COPD vs Healthy: 0.904 & $(2, n=144)$ \\
\hline \multirow[t]{2}{*}{ Interquartile range (ng/ml) } & 1.770 & 1.851 & 1.781 & & \\
\hline & $(3.168-1.397)$ & $(2.702-0.851)$ & $(2.577-0.796)$ & & \\
\hline
\end{tabular}

ILD=interstitial lung disease, COPD=chronic obstructive pulmonary disease, OPN=osteopontin, ng/ml=nanograms per milliliter 
Table 3 Percentage of patients of Interstital lung disease in various subtypes

\begin{tabular}{|c|c|c|c|c|}
\hline \multirow{2}{*}{$\begin{array}{l}\text { Subtype of ILD } \\
(\mathrm{N}=52)\end{array}$} & \multirow{2}{*}{$\begin{array}{l}\text { Percentage } \\
\text { of patients }\end{array}$} & \multicolumn{2}{|c|}{ Serum level of OPN $(\mathrm{ng} / \mathrm{ml})$} & \multirow{2}{*}{$p$-value } \\
\hline & & Median & Interquartile range & \\
\hline Idiopathic pulmonary fibrosis & $50.0 \%$ & 2.173 & 2.020 & H statistic \\
\hline Interstitial pneumonia with autoimmune features & $25.0 \%$ & 2.329 & 2.000 & $=2.389$ \\
\hline Non idiopathic pulmonary fibrosis & $9.6 \%$ & 2.200 & 1.660 & $(4, N=52)$ \\
\hline Hypersensitivity pneumonitis interstitial pneumonias & $11.5 \%$ & 1.462 & 1.02 & $\mathrm{p}$-value $=0.495$ \\
\hline Granulomatous interstitial pneumonias (sarcoidosis) & $3.8 \%$ & 6.881 & - & \\
\hline
\end{tabular}

ILD=Interstitial lung disease, OPN=osteopontin, $\mathrm{ng} / \mathrm{ml}=$ nanograms per milliliter

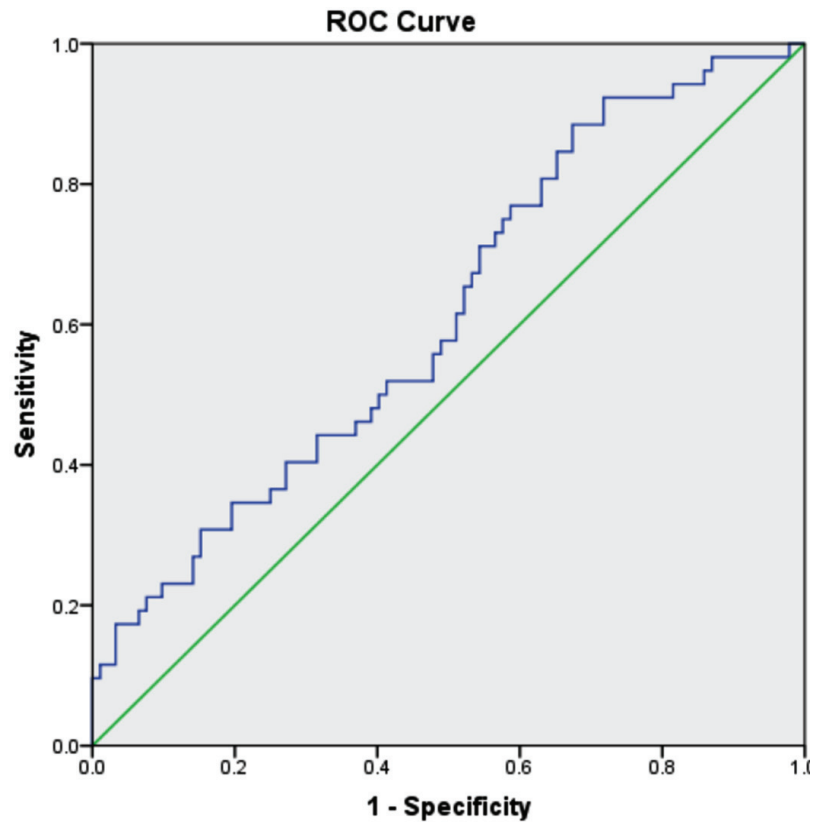

(Area under curve $=0.614, \mathrm{p}$-value $=0.023$ )

$\mathrm{ROC}=$ receiver operating characteristics curve.

Figure 1 Receiver operating characteristics curve of osteopontin levels in patients with interstitial lung disease

\section{Discussion}

ILD is known to be a large spectrum of lung disorders that affects the parenchyma, and is characterized by fibroproliferation and excessive accumulation of extracellular matrix in the lung. It is a progressive disease that does not easily respond to treatment. ${ }^{20}$

With the understanding of the basic cellular processes responsible for pulmonary fibrosis, numerous molecular biomarkers associated with disease progression have been identified, but clinical trials have yet to validate the clinical efficacy of these markers. ${ }^{1}$ Research has revealed that OPN is highly expressed in lung diseases, and directly or indirectly involved in the regulation of granuloma formation and fibrosis. ${ }^{21}$ In an experimental study on mice it was found that after exposure to titanium dioxide, mice not only develop a fibroproliferative lung disease, but also showed increased expression of OPN mRNA; and in turn increased levels of OPN in bronchoalveolar lavage fluid. ${ }^{22}$ In another experimental study, it was found that OPN gene and protein were expressed on the alveolar macrophages in the lung after administration of bleomycin along with the infiltration of inflammatory cells and thickening of alveolar walls, followed by pulmonary fibrosis. ${ }^{6}$ These observations suggested that OPN functions like a cytokine and may be involved in the pathogenesis of fibrotic lung diseases. This present study was thus planned to evaluate the role of serum levels of OPN in the diagnosis of ILD.

The study subjects included 56 diagnosed patients with ILD, who were then compared with 46 patients of COPD, and an equal number of apparently healthy controls. A male preponderance was seen in the patients with ILD 
as well as patients of COPD of the study group (53.8\% and $78.2 \%$, respectively); although this difference in gender was found to be significant only in the patients of COPD. The reason for increased prevalence of the disease in males could be attributed to the increased percentage of exposure of smoke in males as compared to females, considering that smoking is an established risk factor for COPD. These observations are in line with results forwarded in other recent studies. $^{6,23}$

Serum OPN levels were found to be significantly higher in patients with ILD $(2.186 \mathrm{ng} / \mathrm{ml})$, as compared to patients of COPD $(1.68 \mathrm{ng} / \mathrm{ml})$ and healthy controls (1.92 $\mathrm{ng} / \mathrm{ml})$. The results are in concordance with the results of a study performed in a Japanese population, wherein OPN levels were also found to be significantly higher in patients with ILD. The authors found that OPN could be used to discriminate between IP and control subjects. ${ }^{6}$ Another study, from Mexico City of the United States of America, showed that OPN gene expression is highly up-regulated in IPF lungs. They found that the levels of OPN were increased 20-fold in patients with ILD, not only in serum, but also in bronchoalveolar lavage fluids. ${ }^{20}$

In this present study, no significant difference in serum OPN levels was found between patients of COPD and healthy controls. These results are contrary to some earlier published studies, that revealed higher OPN levels in patients of COPD as compared to healthy controls. ${ }^{24-26}$ COPD is characterized by progressive airflow obstruction and excessive inflammation, and OPN levels may be increased in COPD owing to its role in inflammation as a cytokine. However, research has also shown that levels of OPN in COPD increase with severity of the disease and often decrease after improvement. ${ }^{24}$ All patients with COPD in the disease control group of the present study were on treatment and in the stable form of the disease, which could explain the non-significant difference in levels of OPN between the disease controls and healthy controls.
Smoking also affects the inflammatory pattern and in this capacity may be reflected in the levels of OPN. Many studies conducted on patients have found increased serum levels of OPN in smokers as compared to non-smokers. ${ }^{24,26-28}$ In the present study however, no significant difference in levels of OPN was found between smokers, ex-smokers and non-smokers in patients with ILD as well as COPD. Similar results have been forwarded by a study, where it was found that smoking caused concentration dependent increase in OPN levels in cultured endothelial cells. The study also found that in smokers who quit smoking for five days, serum OPN levels were significantly lower compared to those measured prior to cessation of smoking. ${ }^{29}$ The results of another study conducted to evaluate OPN levels in smokers and ex-smokers COPD patients, found significant increased levels in smokers; however, no difference was found between ex-smokers and healthy controls. ${ }^{26}$ As the extent, duration of smoking in smokers and time of cessation of smoking in ex-smokers was not evaluated, this could have a bearing on the non-significant difference in levels of OPN amongst smokers, ex-smokers and non-smoking subjects of the study groups.

Migration and proliferation of fibroblasts are essential for the formation of the fibroblastic foci that seem to represent the corner stone of the progressive fibrotic process in ILD. ${ }^{20}$ The observation that OPN induced both migration and proliferation of primary human lung fibroblasts suggests the sufficiency of OPN secreted from alveolar epithelial cells to function as a fibrogenic cytokine, and induce many of the phenotypic changes associated with lung fibrosis. ${ }^{16}$ The significantly raised levels of OPN in patients with ILD, compared to controls in the subjects of the study group of the present study, support this association of OPN with the pathogenesis of ILD.

Moreover, when the cut-off value was set at 1.089 $\mathrm{ng} / \mathrm{ml}$, based on the ROC curve analysis, the level of OPN in serum reliably distinguished patients with ILD from 
controls ( $p$-value of 0.023 ). OPN levels of more than 1.089 $\mathrm{ng} / \mathrm{ml}$ were found to have a positive predictive value of $42.5 \%$ and a negative predictive value $83.3 \%$, for diagnosis of ILD in the subjects of the study group.

The patients with ILD were categorized into subtypes based on ATS/ERS 2018 classification. ${ }^{18}$ A majority of the percentage of patients with ILD were found to be diagnosed with IPF $(50.0 \%)$, while the least percentage was found to be of granulomatous interstitial pneumonias (3.8\%). The results of a previous study from India showed sarcoidosis as the most common subtype (37.3\%), followed by IPF (27.6\%). ${ }^{30}$ In another study on ILD from India, hypersensitivity pneumonitis was found to be the most common subtype of ILD. ${ }^{31}$ The difference in the prevalence of different subtypes of ILD may be due to different geographical distribution, difference in patient selection and the study design.

Levels of OPN were found to be highest in patients with IPF and IPAF as compared to the other subtypes, although the difference between various subgroups was not found to be significant. The results of another study, however revealed that significantly raised levels of OPN could differentiate between IPF and other ILD's along with markers of extracellular matrix remodelling, such as surfactant protein-D and matrix metalloprotein $7 .{ }^{32}$

The limitations of the present study were the small sample size, due to time and resource constraints. Another limitation, was that the study group was compared with only one disease control group, again due to resource constraints. This present study included all diagnosed cases of ILD attending the out-patient clinic of the department of Pulmonary Medicine during the study period, for whom appropriate subject characterization and sample analysis was done. This has led to the interpretation of the results. However, larger studies with different sets of cohorts may be planned in the future to validate the results, and ascertain the role of OPN in diagnosis and differentiation between different subtypes of ILD.

\section{Conclusion}

The results of the study suggest that serum levels of OPN are increased in patients with ILD, and OPN may be used to aid in the diagnosis of ILD. However, further evaluation with longitudinal studies are warranted in a large population to ascertain these results, and to establish a cause- effect relationship.

\section{Conflict of interest}

The authors declare that there is no conflict of interest regarding the publication of this paper.

\section{References}

1. Ask K, Hambly N, Kolb MRJ. Biomarkers in interstitial lung disease: moving towards composite indexes and multimarkers? Curr Pulmonol Reports 2015;4:125-9.

2. Ley B, Brown KK, Collard HR. Molecular biomarkers in idiopathic pulmonary fibrosis. Am J Physiol Lung Cell Mol Physiol 2014; 307:681-91.

3. Kumar A, Prashant A, Gupta KA, Gautam KA, Kumar A, Choudhri A. Profile of interistial lung diseases at tertiary care centre of northern India. Eur J Pharm Med Res 2016;3:368-74.

4. Hambly N, Shimbori $\mathrm{CH}$, Kolb MA. Molecular classification of idiopathic pulmonary fibrosis: personalized medicine, genetics and biomarkers. Respirology 2015;20:1010-22.

5. Kahles F, Findeisen HM, Bruemmer D, Osteopontin: A novel regulator at the cross roads of inflammation, obesity and diabetes. Molecular Metabolism 2014;3:384-93.

6. Kadota J, Mizunoe S, Mito K, Mukae H, Yoshioka S, Kawakami $\mathrm{K}$, High plasma concentrations of osteopontin in patients with interstitial pneumonia. Respir Med 2005;99:111-7.

7. Sato W, Tomita A, Ichikawa D, Lin Y, Kishida H, Miyake S. CCR2(+) CCR5(+) t cells produce matrix metalloproteinase-9 and osteopontin in the pathogenesis of multiple sclerosis. $J$ Immuno 2012;189:5057-65.

8. Agnholt J, Kelsen J, Schack L, Hvas CL, Dahlerup JF, Sorensen ES. Osteopontin, a protein with cytokine-like properties, is 
associated with inflammation in Crohn's disease. Scandinavian J Immuno 2007;65:453-60.

9. Wong CK, Lit LC, Tam LS, Li EK, Lam CW. Elevation of plasma osteopontin concentration is correlated with disease activity in patients with systemic lupus erythematosus. Rheumatology 2005;44:602-6.

10. Xu L, Ma X, Wang Y, Li X, Qi Y, Cui B. The expression and pathophysiological role of Osteopontin in Graves' disease. J Clin Endocrinol Metab 2011;96:1866-70.

11. Raja R, Kale S, Thorat D, Soundararajan G, Lohite K, Mane A. Hypoxia-driven osteopontin contributes to breast tumor growth through modulation of hif 1 alpha-mediated VEGFdependent angiogenesis. Oncogene 2014;33:2053-64.

12. Tilli TM, Franco VF, Robbs BK, Wanderley JL, Da Silva FR, De Mello KD. Osteopontin-c splicing isoform contributes to ovarian cancer progression. Mol Cancer Res 2011;9:280-93.

13. Waller AH, Sanchez-Ross M, Kaluski E, Klapholz M. Osteopontin in cardiovascular disease: a potential therapeutic target. Cardiol Rev 2010;18:125-31.

14. Psarras S, Mavroidis M, Sanoudou D, Davos CH, Xanthou G, Varela $A E$. Regulation of adverse remodelling by osteopontin in a genetic heart failure model. Eur Heart J 2012;33:1954-96.

15. Hirose M, Tozawa K, Okada A, Hamamoto S, Higashibata Y, Gao B. Role of osteopontin in early phase of renal crystal formation: immunohistochemical and microstructural comparisons with osteopontin knock-out mice. Urol Res 2012;40:121-9

16. Takahashi F, Takahashi K, Okazaki T, Maeda K, Lenaga H, Maeda M. Role of osteopontin in the pathogenesis of bleomycininduced pulmonary fibrosis. Am J Respir Cell Mol Biol 2001;24: 264-71.

17. Zuo F, Kaminski N, Eugui E, Allard J, Yakhini Z, Ben-Dor A et al. Gene expression analysis reveals matrilysin as a key regulator of pulmonary fibrosis in mice and humans. Proc Natl Acad Sci 2002;99:6292-7.

18. Cottin V, Hirani NA, Hotchkin DL, Nambiar AM, Ogura T, Otalo M, et al. Presentation, diagnosis and clinical course of the spectrum of progressive fibrosing interstitial lung diseases. Eur Respir Rev 2018;27:180076.

19. Global Initiative for Chronic Obstructive Lung Disease. Global Strategy for the Diagnosis. Management and Prevention of COPD [monograph on the Internet]. Fontana: GOLD; 2017 [cited 2020 May 25]. Available from: https://goldcopd.org/wp-
content/uploads/2017/11/GOLD-2018-v6.0-FINAL-revised20-Nov_WMS.pdf

20. Pardo A, Gibson K, Cisneros J, Richards TJ, Yang Y, Becerril $C$, et al. Up-regulation and profibrotic role of osteopontin in human idiopathic pulmonary fibrosis. PLoS Med 2005;2:891903.

21. O'Regan A. The role of osteopontin in lung disease. Cytokine Growth Factor Rev 2003;14:479-88.

22. Mangum JB, Bermudez E, Sar M, Everitt Jl. Osteopontin expression in particle-induced lung disease. Exp Lung Res 2004;30:585-98.

23. Ashizawa N, Graf K, Do YS, Nunohiro T, Giachelli CM, et al. Osteopontin is produced by rat cardiac fibroblasts and mediates $\mathrm{A}(\mathrm{II})$-induced DNA synthesis and collagen gel contraction. J Clin Inves 1996;98:2218-27

24. Ma JE, Lee SH, Kim YE, Lim SJ, Lee SJ, Jeong YY, Kim HC, Lee JD, Hwang YS, Cho YJ. Comparison of serum osteopontin levels in patients with stable and chronic obstructive pulmonary disease and exacerbation. Tuberc Respir Dis 2011;71: 195-201.

25. Papaporfyriou A, Loukides S, Kostikas K, Simoes DC, Papatheodorou G, Konstantellou E et al. Increased levels of osteopontin in sputum supernatant in patients with COPD. Chest 2014;146: 951-8.

26. Ali AA, Azab NY, El-Mahalawy II, Tayel SI, Khalifa EM. Osteopontin in chronic obstructive pulmonary disease: Smokers and ex-smokers. Egypt $\mathrm{J}$ Chest Dis Tuberc 2015;64:63-6.

27. Hillas G, Loukides S, Kostikas K, Simoes D, Petta V, Konstantellou $E$, et al. Increased levels of osteopontin in sputum supernatant of smoking asthmatics. Cytokine 2013;61:251-5.

28. Petta V, Loukides S, Kostikas K, Papaioannou Al, Papatheodorou G, Cholidou K, et al. Serum osteopontin in patients with lung cancer and chronic obstructive pulmonary disease: does the co-existence make the difference?. J Thorac Dis 2018;10:740-8.

29. Bishop E, Theophilus EH, Fearon IM. In vitro and clinical studies examining the expression of osteopontin in cigarette smoke-exposed endothelial cells and cigarette smokers. BMC Cardiovasc Disord 2012;12. doi: 10.1186/1471-2261-1275.

30. Kumar R, Gupta N, Goel N. Spectrum of interstitial lung disease at a tertiary care centre in India. Pneumonol Alergol Pol 2014; 82:218-26. 
31. Singh S, Bridget FC, Sharma BB, Joshi JM, Talwar D, Katiyar $\mathrm{S}$, et al. Interstitial lung disease in India: results of prospective registry. Am J Resp crit care med 2017;195:801-13.

32. White ES, Xia M, Murray S, Dyal R, Flaherty CM, Flaherty
$\mathrm{KR}$, et al. Plasma surfactant protein -D, matrix metalloproteinase -7 and osteopontin index distinguishes idiopathic pulmonary fibrosis from other idiopathic interstitial pneumonias. Am J Respir Crit Care Med 2016;194:1242-51. 